\title{
High Throughput Image Analysis on PetaFLOPS Systems
}

\author{
Robert Henschel, Matthias Müller ${ }^{1}$, and Yannis Kalaidzidis ${ }^{2}$ \\ ${ }^{1}$ Technische Universität Dresden, \\ Zentrum für Informationsdienste und Hochleistungsrechnen (ZIH), \\ 01062 Dresden, Germany \\ Robert.Henschel@zih.tu-dresden.de \\ http://www.tu-dresden.de/zih \\ 2 Max Planck Institute of Molecular Cell Biology and Genetics, \\ Pfotenhauerstr. 108, \\ 01307 Dresden, Germany
}

\begin{abstract}
Today's state of the art high-throughput screening facilities can produce tens of thousands of images of cells per day. Analyzing images from high-throughput screening experiments is very time consuming and computationally demanding. Researchers are currently limited not by the availability of experimental data, but by the computing resources for the image analysis. The Max Planck Institute of Molecular Cell Biology and Genetics Dresden, Germany, (MPI-CBG) and the Center for Information Services and High Performance Computing at the Technische Universität Dresden (ZIH) are working together to integrate high performance computing systems into the workflow of biologists. The MPI-CBG has developed software that biologists use for their image analysis work. The software can utilize local workstations and remote HPC systems for image analysis. Currently the software is used successfully on small clusters and PC-Farms. Most parts of the image analysis workflow of screening experiments can be performed in parallel and is ideal for distribution on large systems. With a few modifications and a new approach to data management, the software should be able to scale to PetaFLOPS systems.
\end{abstract}

\section{High Throughput Image Analysis}

Determining the DNA sequence and the genes of an organism is an automated process. The DNA of a number of organisms has been successfully sequenced and is now available for further research. In contrast, assigning a function to a gene is far more complicated and involves a lot of human interaction.

High-Throughput screening experiments are used to search for gene functions. These experiments do also include a large number of control experiments to cope with off-target effects and other side-effects that occur in complex screening experiments. Because of the large number of images that high-throughput screening experiments produce, automated image analysis software is used to analyze the images and detect phenotypes. 
The research group of Professor Dr. Marino Zerial at the MPI-CBG develops its own image analysis software called Motion Tracking. The project was started because there was no standard software package available on the market that would suit the needs of the biologists, in terms of ease of use, feature set and scalability. The application that is developed at the MPI-CBG is used for the whole image analysis process and despite its name, is not limited to just motion tracking. It is capable of performing image analysis on a large number of images in batch mode. It can also visualize the input and the result data as well as perform statistical calculations. To decrease the overall runtime of image analysis tasks, the application can distribute the calculation on small clusters of workstations running Microsoft Windows or on IA32/AMD64 HPC systems running Linux. Motion Tracking is successfully used in the analysis of current experiments such as research in endocytosis and endosomes [4].

The general image analysis workflow for screening experiments at the MPICBG is show in fig. 11. It consists of six distinct phases. At first, the biological experiment is prepared in plates with, for example, 96 or 384 wells. Each of those wells contains a slightly different experiment, for example different knocked down genes or a control experiment. Depending on the objective of the experiment, the number of required wells can vary quite a lot. In a small screening experiment that is only concerned with 120 genes, 12 96-well plates are required. For a genome wide screening experiment, more than 200384 -well plates would be required. In the next step, confocal images of the cell cultures or single cells are taken with an automated microscope. The microscope acquires images at different positions in each well and stores them in image packages.

In the preprocessing phase the images are extracted from the image packages, separate images for the red, green and blue color channel are combined into one image, out of focus images are removed and images that show no or only very few cells are filtered out. This phase is done in parallel for all images on a small cluster of eight dual XEON workstations at the MPI-CBG. The small cluster is used because the actual compute phase is very small and a lot of $\mathrm{I} / \mathrm{O}$ operations are performed. Since the small cluster can be accessed faster than the $\mathrm{HPC}$ system at the ZIH and a more efficient communication protocol is used for

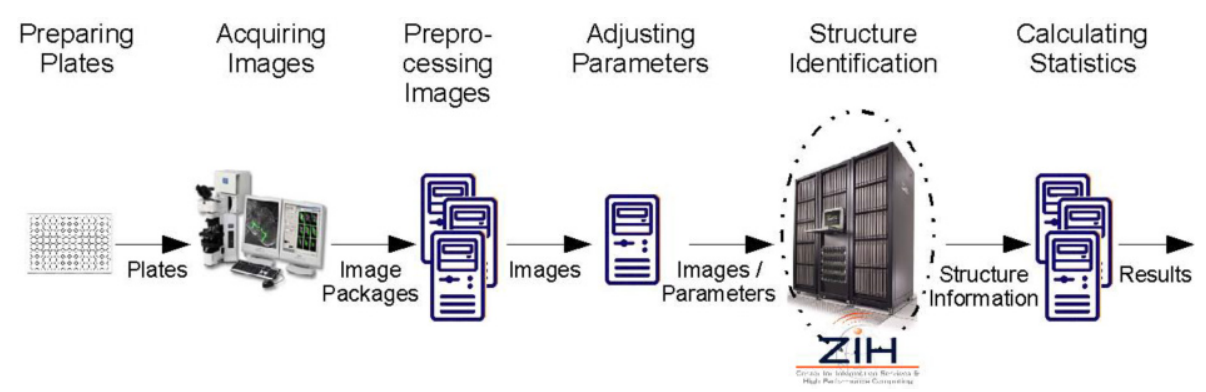

Fig. 1. Image Analysis Workflow at the MPI-CBG 
communication to the small cluster, this phase is performed fastest on the small cluster.

In the next phase, the parameters for the structure identification algorithm are adjusted. Since the optimal parameter values vary between individual image sets, for example depending on the characteristics of the microscope that was used for the screening, this step requires human interaction.

With the parameter file created in the previous phase, all images are searched. Since most analysis tasks have no dependency on images besides the one that is analyzed, this process can be performed in parallel. The image files and the parameter file are transferred to the HPC system and analyzed. This phase is the most computational intensive. The analysis time for a single image can range from a few minutes to one or two hours depending on the analysis that is performed. The structure information is transferred back to the main workstation after the analysis.

The statistics calculation represents the final analysis step that provides answers to the underlying biological question of the screening experiment. It is done on the MPI-CBG cluster again since the calculation time is very small compared to the overhead that is incurred by transferring input files and results to the HPC system at the ZIH and back.

\section{Performance and Limitations}

Motion Tracking is written using a combination of the programing languages $\mathrm{C}++$ and PLUK. The performance critical parts of the application as well as the PLUK interpreter are written in $\mathrm{C}++$ while large parts of the application, the graphical user interface and utility functions are written in PLUK. PLUK is an interpreted programing language that was developed in the mid to late 1990s at the Lomonosov Moscow State University [3]. It was created as a fourth generation programing language, designed to make prototyping of algorithms easy and aid rapid application development. PLUK is not meant as a high performance computing tool, but is targeted at users that need to test algorithms and develop small to medium applications for example to process and analyze data of experiments.

Motion Tracking was developed as a tool for biologists to work with images from screening experiments. When the experiments got larger, it was enhanced to distribute calculation tasks among workstation to shorten the overall analysis time. At that time, the front-end of the application and the underlying PLUK library were only available for Microsoft Windows 2000 and later versions. This meant that the calculation tasks could only be distributed to workstations running a compatible version of Microsoft Windows. For the communication between the calculation workstations and the master workstation, a custom protocol was developed that is implemented using the Winsock library. One communication channel per workstation is used to dispatch tasks and to send and receive data. This channel is kept open for the entire runtime of a task. The solution works 
nicely for a limited number of workstations. The MPI-CBG uses this on a local cluster of 8 dual XEON workstations.

As the experiments got larger, the need for more compute resources arose. The ZIH provided access to its HPC systems, a PC-Farm with 64 dual Opteron nodes and an SGI Altix 3700 system with 192 Itanium 2 CPUs. For Motion Tracking to utilize those systems, the PLUK interpreter and associated libraries were ported to Linux. The graphical user interface that controls the calculation remained on the Microsoft Windows platform. The communication channel was replaced with an implementation that uses the OpenSSH binaries on the Windows platform to perform file transfer and remote job submission. The current version works satisfactory on the PC-Farm with Opteron CPUs. To use the Altix HPC system, the PLUK interpreter must be adapted to the memory alignment requirements of the Itanium $2 \mathrm{CPU}$ which is still an open issue.

The PC-Farm at the ZIH was added to the image analysis workflow with as little modifications to existing software as possible. This means that the existing communication pattern was adapted to work over SSH connections but not really changed to reflect the properties of the new connection. The communication speed to the PC-Farm at the ZIH is slower than to the local workstations at the MPI-CBG. The latency and the startup time for a file transfer are a lot higher as well. To start a job on the PC-Farm, all input data has to be moved to the PC-Farm and a job has to be submitted to the batch system. At the end, the results of the calculation have to be moved back to the MPI-CBG for visualization in Motion Tracking. The input data and the results for each job are in the order of a few megabytes. All this contributes to a very high startup time for a calculation that can only be compensated by running large calculations.

Equation 1 can be used to calculate the number of processors $(n)$ that can be used in parallel on the PC-Farm. If not enough jobs can be submitted from the master workstation at the MPI-CBG to the HPC system at the ZIH, not all available processors can be utilized. The maximum number of processors that can be utilized depends on the average runtime $\left(T_{R}\right)$ of an image analysis job, the number of communication channels $(c)$ that are available to send data and the transfer time per image $\left(T_{I}\right)$ using such a channel. Every communication channel is used on the master workstation by a thread. Every thread prepares the input data, sends it to the HPC system and submits a job to the batch system. Currently, the number of threads is set to 7 . It is limited by the number of concurrent open SSH connection to the HPC system and the load that those connections induce on the master workstation.

The transfer time per image varies between 4,5 and 5 seconds. It can be calculated using (2). The preparation time $\left(T_{P}\right)$ is required to transfer the image to the master workstations memory and repackage it for sending it to the HPC system. The actual transfer time is comprised of the time to open the connection $\left(T_{O}\right)$ and the time to transfer the image, which can be calculated by dividing the image size $\left(I_{S}\right)$ by the transfer rate $(R)$ of the channel. The time to submit a task to the batch system is denoted with $T_{S}$. Values for the individual times can be found in table 1 . 
Table 1. Key parameters of image Analysis jobs

\begin{tabular}{|l|l|}
\hline Item & Value \\
\hline Average runtime of image analysis jobs & $\approx 1800$ seconds \\
\hline Number of channels & 7 \\
\hline Transfer time of one image & $\approx 4.75$ seconds \\
\hline Preparation time & $\approx 2$ seconds \\
\hline Time to open a connection & $\approx 0.5$ seconds \\
\hline Image size & $2 \mathrm{MiB}$ \\
\hline Transfer rate & $\approx 1.6 \mathrm{MiB} /$ seconds \\
\hline Submission Time & $\approx 0.5$ seconds \\
\hline
\end{tabular}

$$
\begin{gathered}
n=T_{R} * \frac{c}{T_{I}} \\
T_{I}=T_{P}+\left(T_{O}+\frac{I_{S}}{R}\right)+T_{S}
\end{gathered}
$$

To utilize the full PC-Farm with $128 \mathrm{CPUs}$, the average runtime of an image analysis job must be at around 90 seconds. If the runtime is shorter the entire PC-Farm will not be filled with jobs before the first job has finished and makes the CPU available again. If the runtime is longer, jobs will pile up in the batch queue and are ready to be dispatched as soon as a CPU becomes available. Fig. 2 shows that the workflow software is able to utilize basically all available CPUs of the $128 \mathrm{CPU}$ cluster.

Image analysis jobs are always longer than 90 seconds, so the overhead of the network transfer is no problem. The runtime of jobs in the preprocessing phase is largely determined by $\mathrm{I} / \mathrm{O}$ operations of reading image packages and writing single images. This phase is not done on the PC-Farm. The runtime of jobs in the statistical calculation phase depends on how many statistical calculations are performed per image and how complex they are. The more calculations are performed, the better is the chance that such a job can be run efficiently on the PC-Farm.

Overall, the current solution works nicely for the current size of the PC-Farm. However, when the PC-Farm is upgraded to 2500 Opteron cores the submission rate has to be increased to be able to utilize the whole PC-Farm. If the submission rate stays at 1.5 jobs per second, the average job length would have to be more than 1300 seconds to utilize the whole PC-Farm.

\section{Suggested Changes to Scale Up to PetaFLOPS Systems}

None of the limitations outlined above are due to the underlying image analysis workflow, they exist because of the current implementation. The current version of Motion Tracking has grown over time and was successfully adapted to new requirements. With the growing number of images that screening experiments produce, not only Motion Tracking must be adapted, but also the underlying storage concept must be changed to better fit the workflow. 


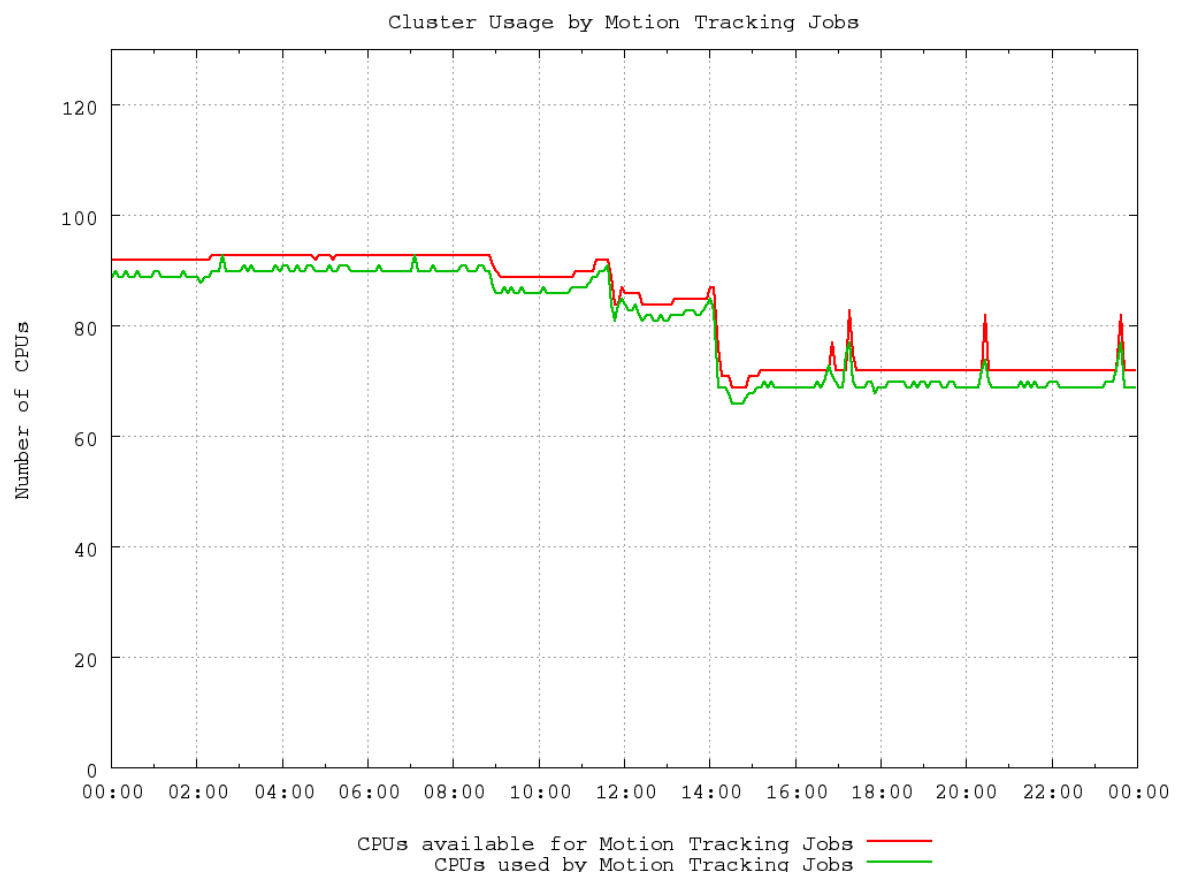

Fig. 2. Available and used CPUs during one day

In the structure identification phase, all images could be analyzed in parallel as there are no dependencies between images. The degree of parallelism is only limited by number of available CPUs and the ability to move the required input data to those CPUs. The same is true for the statistical calculation phase only that the computing time is generally shorter in that phase. To achieve such a high degree of parallelism, a number of changes would have to be implemented.

\subsection{Introduce Distributed Storage of Data}

In the current version, all data is stored at a central location and is only transferred to the HPC system for the period of the analysis. When the structure identification phase needs to be repeated, because different parameters are selected, the raw images have to be transfered again from the MPI-CBG to the ZIH. This can be avoided by introducing a resource broker or a global database that can be queried for the location of particular image files.

Also, the results that are created in the structure identification phase are the input data for the statistical calculation phase. Currently the results are transferred back to the MPI-CBG because they are needed for further analysis that can either be performed in parallel on a cluster or by the master workstation alone. Also, for exporting results they have to be available locally at the MPICBG. 
Raw images and the result files could be replicated between both locations. That would guarantee fast access and availability on both locations. For this approach to be successful, a layer that takes care of data management, such as tracking and replicating images, must be added.

\subsection{Improve Communication and Job Control}

Currently, the communication with the HPC systems is built around the OpenSSH binaries for transferring files and executing remote commands. This implementation was choosen to support as many Linux based HPC systems as possible. The solution is very flexible and can be adapted quickly to different environments.

The disadvantages are limited transfer rates and only basic job control and status information about running jobs on HPC systems. When using Windows workstations as compute resources, Motion Tracking uses a custom communication protocol that allows for example to query jobs status information. This communication protocol is not yet ported to Linux.

To increase the transfer rates $\mathrm{ssh} / \mathrm{scp}$ can be replaced with mechanism like GridFTP 1, that provide higher utilization of the available network bandwidth. To improve job submission and control on large HPC systems, the use of grid middle-ware such as UNICORE[5] or Globus [2] as alternative to the proprietary protocol of Motion Tracking will be examined.

With the above mentioned changes in place, Motion Tracking should be prepared to benefit from large HPC systems.

\section{References}

1. W. Allcock, J. Bresnahan, R. Kettimuthu, and M. Link. The globus striped gridftp framework and server. In SC'05: Proceedings of the 2005 ACM/IEEE conference on Supercomputing, 2005.

2. Ian Foster. Globus toolkit version 4: Software for service-oriented systems. $J$. Comput. Sci \& Technol., 21(4):513-520, July 2006.

3. Y.L. Kalaidzidis, A.V. Gavrilov, P.V. Zaitsev, A.L. Kalaidzidis, and E.V. Korolev. PLUK - an environment for software development. Programming and Computer Software, 23(4):206-212, 1997.

4. J. Rink, E. Ghigo, Y. Kalaidzidis, and M. Zerial. Rab conversion as a mechanism of progression from early to late endosomes. Cell, 122(5):735-749, 2005.

5. A. Streit, D. Erwin, Th Lippert, D. Mallmann, R. Menday, M. Rambadt, M. Riedel, M. Romberg, B. Schuller, and Ph Wieder. Unicore - from project results to production grids, 2005 . 